\title{
VLSI Design of A Chip With High Speed Atm Switch-A Review
}

\author{
Vishal Chandra $^{a}$, Dr. Probodh Khampariya ${ }^{b}$ \\ a Research Scholar, Dept. Electronics and Communication Engineering, \\ Sri Satya Sai University of Technology \& Medical Sciences, Sehore, Bhopal Indore Road, Madhya Pradesh, India \\ b Research Guide, Dept. of Electronics and Communication Engineering, \\ Sri Satya Sai University of Technology \& Medical Sciences, Sehore, Bhopal Indore Road, Madhya Pradesh, India
}

Article History: Received: 11 January 2021; Accepted: 27 February 2021; Published online: 5 April 2021

\begin{abstract}
In current computer communication network, it is overwhelmed by two technologies, in particular Asynchronous Transfer Mode (ATM) and Internet Protocol (IP). Association situated ATM is the awesome constant administrations which require ensured nature of-administration like video conferencing. Be that as it may, connectionless IP is more proficient than ATM for non-ongoing administrations like email. Right now, the significant exploration challenge is on the most proficient method to coordinate ATM and IP into a solitary network effectively. It is shown by the acknowledgment of the highlight of the A/I Net architecture: the A/I Switch. In this postulation, a VLSI execution of a multistage self-steering ATM switch texture which is one of the vital parts of the A/I Switch will be presented. The size of the switch model is 16x16. The chip is intended to work at the very least frequency of $100 \mathrm{MHz}$ and the framework is equipped for dealing with the OC-12 (622 Mbps) connect rate. In view of a piece cut architecture, the whole 16x16 switch is acknowledged utilizing four indistinguishable chips. It accomplishes elite by using dispersed control and accelerate with the input-output buffering technique. A need structure, which upholds four-level, permits the postponement delicate ATM cells to be switched with the briefest inertness. It likewise empowers the non-interleaving directing plan of IP cells.
\end{abstract}

\section{Introduction}

Communication network limit and applications have been changing at a huge rate for as far back as twenty years. Other than high velocity, some real-time applications, for example, videoconferencing, music on interest, and video on interest expect messages to be shipped off more than one objective. Therefore, supporting multicast has become a vital prerequisite for any switch intended for future broadband communication networks.

ATM-like fixed-sized bundle switching draws in much interest in light of its application in cutting edge Internet switches and switches. A variable-sized approaching IP bundle is inside sectioned into fixed-size ATM-like cells which are switched to the output ports, where they are reassembled into the IP datagram. Getting from ATM wording, we utilize the term cell to distinguish the fixed-sized bundle utilized in the switch, which can be ATM cells or some other helpful information design.

Multistage interconnection network (MIN) plan has become an appealing answer for broadband switch architecture because of the numerous alluring highlights, for example, self-directing, dispersed control, particularity, steady deferral for all input-output sets and reasonableness for VLSI usage. A multicast switch texture utilizing implied cell replication is favored in light of the fact that it joins the steering and replication capacities into a solitary bound together network. This sort of configuration will acquire the majority of the alluring highlights of the MIN plan.

\section{Switching}

A switch is a network component that transfers bundles from input ports to the fitting output ports. Here, a port alludes to a state of connection in the switch. Appropriately, switching is a cycle of transfer of bundles from input ports to output ports. This transfer is additionally alluded to as inside directing of parcels. Switching or inner steering structures bite the dust center elements of the switch. A switch with equivalent number of input and output ports is known as a symmetric switch. The two center switching issues are inside directing and buffering.

\section{ATM Switch Design Issues}

ATM has arisen as the favored transfer mode for fast transmission of information. This advancement is the consequence of supported examination in the field of switching over a time of one and a half many years. The Endeavor isn't just to create switches, equipped for switching a large number of cells each second yet additionally to ensure the QoS (Quality of Service) guarantees of ATM innovation. All things considered, effective switching and productive traffic the board of cells hold the way to conveying the QoS guarantees of ATM. The way that ATM is a cross breed of conventional circuit switching (for voice transfer) and bundle switching (for information transfer) has a significant bearing on ATM switching architectures. Be that as it may, neither bundle switching nor circuit switching architectures are straightforwardly appropriate to ATM switches.

\section{Performance Measures in Switch design}

The important issues to be considered while designing an ATM switch are

Design complexity

Packet loss probability

Throughput

Cost 
Delay

Scalability

\section{Typical ATM Switch}

An ATM switch is something other than an interconnection structure that cushions and courses cells. Actually, the control plane capacities and traffic the executives elements of an ATM switch present huge plan intricacy. Figure 2 portrays an average switch and Figure 1 shows the elements of an ATM switching unit. As seen from the Figure, a switch has three essential parts.

The three core modules of an ATM switch are

Input Module

Switching fabric

Output Module

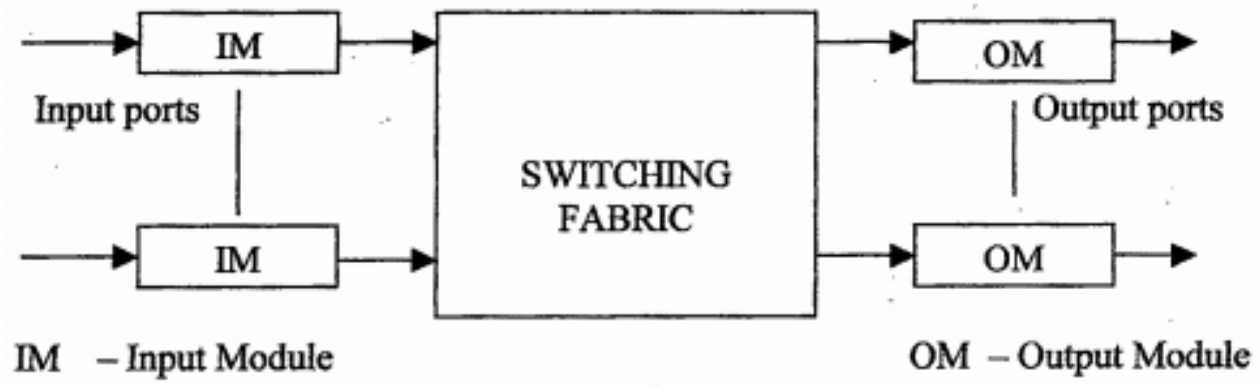

Figure 1. Typical ATM switch

\section{Literature Review}

Udriyah et al., The up to date and the efficient advertisement of products and services enable the firms to broaden the scope of marketing. Different efficient tools are used for advertisement. The efficient advertisement clearly defines the features and quality of products; it clarifies its channels to purchase the products and communicates about its policies about the prices of products. China Unicom also adopts promotional strategies to advertise its products and promote their marketing. For instance, it uses catalogs, runs tradeshows, and mail campaigns to introduce its products. Research has proved that the Innovation based and efficient advertisement process is playing a key role in marking of China Unicom's products. The telecom network consists of such parts as transport, multiplexing, switch and terminal. Among them, the three parts of transport, multiplexing and switch combined are known as "transfer mode". ATM stands for Asynchronous Transfer Mode, which integrates the strength of both circuit switch and packet switch, i.e. both flexible bandwidth allocation and elimination of complex traffic control and error control. This greatly reduces the time delay in transport and is qualified for LAN interconnection to provide real-time multimedia services with QOS guarantee. ATM is a connectionoriented communication mode. Connection orientation refers to the establishment of a connection between the sender and receiver before communication, and the message or information is continuously transferred on that connection while in communication. Therefore the routing between the sender and receiver is fixed for multiple messages or information in a single communication. ATM is a packet switch mode with fixed packet length. In traditional packet switching the length is not fixed. ATM relies on cell switching technology. ATM offers statistical multiplexing capability. ATM can integrate multiple services.

Anuradha, et al., ATM (Asynchronous Transfer Mode) network is connection-oriented high speed multiservice network. Now a days ATM networks are used as backbone networks in Telecom industry. ATM network supports different service classes, include ABR (Available Bit Rate), rt-VBR (real time Variable Bit Rate), nrtVBR (non-real time Variable Bit Rate), CBR (Constant Bit Rate), UBR (Unspecified Bit Rate), and GBR (Guaranteed Bit Rate)to handle multiple services like data, voice, video and so on. But burtsy traffic handled by ABR, rt-VBR, nrt-VBR, GBR service classes, but all have their own limitations. This paper presents an overview of different Traffic shaping, Congestion Control mechanisms to handle bursty traffic in ATM networks.

Md. Main Uddin et al., Every year our local banks spend a lot of money for installing and maintaining switching software provided by foreign vendors. Is it possible to implement ATM/POS switch software in Bangladesh cost effectively? We did requirement analysis of implementing ATM/POS switching software. We tried to find out shortcomings of implementing ATM/POS switching software in Bangladesh. We did survey in 2 Banks IT sector and talked with Vendor of Switching software to study details of switching software. Finally we tried to estimate manpower and time needed to implement Switching software in Bangladesh.

Sheikh et al., this paper presents an optimal proportional bandwidth allocation and data droppage scheme to provide differentiated services (DiffServ) for downlink pre-orchestrated multimedia data in a single-hop wireless network. The proposed resource allocation scheme finds the optimal bandwidth allocation and data drop rates under minimum quality-of-service (QoS) constraints. It combines the desirable attributes of relative DiffServ and 
absolute DiffServ approaches. In contrast to relative DiffServ approach, the proposed scheme guarantees the minimum amount of bandwidth provided to each user without dropping any data at the base-station, when the network has sufficient resources. If the network does not have sufficient resources to provide minimum bandwidth guarantees to all users without dropping data, the proportional data dropper finds the optimal data drop rates within acceptable levels of QoS and thus avoids the inflexibility of absolute DiffServ approach. The optimal bandwidth allocation and data droppage problems are formulated as constrained nonlinear optimization problems and solved using efficient techniques.

Ibrahim et al., proposed a two phase GA for VLSI test vector selection method which outperforms other proposed heuristic algorithms in different scenarios, while taking into consideration the conditions priorities and computing cycles required by each test vector. Results show that intelligent selection of test vectors achieve target coverage using the minimum number of computing cycles.

Skobtsov et al., presented two ways of TPG for VLSI circuits. One method is based on the classical GA and other method includes genetic programming, in which test patterns are represented as a micro operation sequence. In this, linear graph representation is used for the representation of patterns and their related operations i.e. crossover and mutation.

Krishna et al., proposed low power pseudo exhaustive testing technique with CA. In this work a seed value is identified for CA that dissipates the minimum energy during test proposed a reordering algorithm for minimizing the test power in VLSI circuits by calculating the reordered test vectors for minimum total Hamming distance. They performed experiments on ISCAS benchmark circuits and reported $21 \%$ reduction in switching activity in comparison with conventional ATPG algorithms.

Duan et al., proposed a bus encoding technique using forbidden transition free algorithm for cross-talk reduction for on-chip VLSI interconnect. The mapping and coding scheme was proposed based on a variant of binary Fibonacci number system. The mathematical analysis showed that all numbers can be represented by Forbidden Transition Free (FTF) vectors in the Fibonacci Numeral System (FSN) which reduced crosstalk delay and gave a recursive procedure to generate crosstalk delay free binary Fibonacci code words.

Xia et al., The total bandwidth leased for carrier operations in Asynchronous Transfer Mode ("ATM") and Frame Relay ("FR") amounted to 9007 x 2 Mbps. The broadband videotelephony service "Uni-Video" terminals accumulated at 337thousand. The Business offered the "Uninet" Internet access service in 328 cities in China as of 31 December 2004, providing "Uninet" regional roaming service with 103 countries and regions. Internet subscribers rose from 12.43 million in 2003, to 13.62 million. By 31December 2004, "Ruyi Mailbox" subscribers had reached 14.69 million.

Minje et al., discussed about reducing the number of crossbar links between the input and output lines. This can be done by merging the entire cross bars and removing the redundant once. Iterate the process of merging the cross bars and removing the redundant one until there is no repetitive links between the input and the output lines. This cascading is done before the routing of packets from source to destination begins.

En-Jui et al., said that the level of congestion is not known exactly, when the calculation for congestion is taken from the status of channels alone. The status of switches is also needed to find the exact status of congestion. The switch congestion level can be calculated with the rate of change of the buffer level. By adding the channel congestion and the switch congestion the actual status or the waiting time of packets can be calculated.

Yi and Sudeep et al., represents a promising solution to overcome the worsening communication performance of the electrical wires is Silicon Nanophotonics. This can be done by transferring data using light signaling between cores and memory, orders of magnitude improves the bandwidth, latency and energy are possible. Yi and Sudeep present a tutorial regarding the possibilities and challenges of photonic network-on-chip as the communication backbone for multicore chips.

Ramani et al., took a case study for optimizing the latency for NoC systems. NoC gives a good scaling and freedom from the complex wiring. The five different routing architectures namely, low latency router by Look ahead adaptivity, NoC router with look ahead Bypass, Look ahead routing algorithm for 3DNoC architecture, Wormhole switching architecture and Connectionoriented service in MANGO clock less NoC are compared for Best effort routing and Guaranteed Services, which in turn increases the Jitter as well.

Kunj et al., made a survey on arbitration for NoC. Arbiter, a prime component has a great impact on the viability of router. The study has been done to overcome the extensive problems of starvation, HOL, congestion, etc. The arbiter comparison has been made between the following arbiter types, Fixed Priority Arbiter, RoundRobin Arbiter, Matrix Arbiter, Tree Arbiter, Multi-Priority Arbiter, Dynamic Adaptive Arbiter (DDA), Hierarchical Round Robin Arbiter, Ring Arbiters, Square Arbiter, Token Ring Arbiter and Lottery Arbiter. The goal of designing congestion free, low latency, high speed scheduler is to use DAA as the base of operation is concluded in this survey.

Paolo Meloni et al., The paper presented a methodology for characterization of NoC switch area and power requirements. Their tests show that, at any rate at the $0.13 \mu \mathrm{m}$ hub, applying the strategy to netlist level gadgets yields a satisfactory estimate of the genuine conduct even after position and directing, however that considerably more prominent accuracy can be accomplished, whenever wanted, by applying a similar procedure at the format 
level. For their situation they have picked, the rectangular changes to add a more modest measure of data to the preparation set. Their primer interior testing affirms this property, at any rate for the Xpipes NoC.

Andreas Hansson et al., In this paper the problem of mapping cores onto any given NoC topology and statically route the communication between these cores is considered. They show how the pruning and the cost metric utilized in way determination can be stretched out past one channel to catch the idea of virtual circuits. Gathering introduced the UnMappable Read Architecture (UMARS+) calculation which coordinates the spatial planning of centers, three asset allotment stages, spatial steering of correspondence and TDM schedule opening task. They applied the calculation to a Moving Picture Expert Group (MPEG) decoder SoC, improving territory 33\%, power scattering $35 \%$ and worstcase dormancy by a factor of four over a customary cascade approach. They have indicated how an exceptionally adaptable turn forbiddance calculation can be utilized to give most extreme addictiveness in steering of best exertion streams. The time unpredictability of UMARS+ is low and trial results show a run time just $20 \%$ higher than that of way determination alone. As the primary commitment, they have demonstrated that how the planning can be completely fused in way choice. The proposed calculation depends on the preclusions on remaining assets with the end goal that best exertion streams can utilize what isn't needed by ensured administration streams. By the fuse of the crossed way in cost counts, they inferred a metric that reflects how reasonable a channel is when utilized after the channels previously navigated.

\section{Conclusion}

The present chip executes another architecture that has a few significant focal points. It is proficient away space like a common memory switch and versatile in size like a space division switch. The measure of buffering and thus the phone misfortune can be changed by the expansion of additional dissemination layers. The architecture is proficient in the quantity of layers required and subsequently requires generally couple of chips when contrasted with other space-division switches, for example, the rerouting banyan network which may require at least 50 layers of switching hubs. Computer reenactments have affirmed that the architecture jelly cell grouping and it is proficient as far as storage space.

\section{REFERENCES}

1. Jean Yves Le Boudec, "The Asynchronous Transfer Mode: a Tutorial," Computer Networks and ISDN Systems, The International Journal of Computer and Telecommunications Networking, vol. 24, no. 4, 1992, pp.279-309.

2. Paul Shipley, Magdy Bayoumi, "A Scaleable Multibuffer ATM Switch Architecture", Proceedings of the ISCA International Conference on Parallel and Distributed Computing, 1995, pp. 313-317.

3. H. Jonathan Chao, Byeong-Soeg Choe, "A Large-scale Multicast Output Buffered ATM Switch", Globecom 93, pp. 34-41.

4. A. Jajszczyk and W. Kabacinski, "A Growable Shared-BuEered-Based ATM Switching Fabric", pp. 29-38, Proceedings of Globecom 93.

5. Ambeth Kumar, V., Malathi, S., Venkatesan, R., Ramalakshmi, K., Vengatesan, K., Ding, W., \& Kumar, A. (2020). Exploration of an innovative geometric parameter based on performance enhancement for foot print recognitionJournal of Intelligent \& Fuzzy Systems(Preprint), 1-16

6. Sheikh, Waseem \& Ghafoor, Arif. (2010). An optimal bandwidth allocation and data droppage scheme for differentiated services in a wireless network. Cyber Center Publications. 10. 10.1002/wcm.779.

7. Ibrahim, W., El-Chouemi, A., and Amer, H. (2006). "A Two-phase Genetic Algorithm for VLSI Test Vector Selection”. In IEEE Congress on Evolutionary Computation, pages 878-884. IEEE.

8. Skobtsov, Y., Ivanov, D., Skobtsov, V., Ubar, R., and Raik, J. (2010). "Evolutionary Approach to Test Generation for Functional BIST". arXiv preprint arXiv:1008.0063.

9. Krishna, K., Uday, B., and Chattopadhyay, S. (2009). "Low Power Pseudoexhaustive Testing with Cellular Automata". In International Conference on Advances in Computing, Control, \& Telecommunication Technologies, 2009. ACT'09., pages 419-423. IEEE.

10. Duan, C., Zhu, C., and Khatri, S. P. (2008). "Forbidden Transition Free Crosstalk Avoidance CODEC Design". In Proceedings of the 45th annual Design Automation Conference, pages 986991. ACM.

11. Udriyah, Tham J., Azam, S. M. F. (2019). The effects of market orientation and innovation on competitive advantage and business performance of textile SMEs, Management Science Letters

12. Xia, J. (2011). The third-generation-mobile (3G) policy and deployment in China: Current status, challenges, and prospects. Telecommunications Policy, 35(1), 51-63.

13. Therdpong DAENGSI et.al, IP Telephony: Comparison of Subjective Assessment Methods for Voice Quality Evaluation, Walailak J Sci \& Tech 2014; 11(2): 87-92.

14. VEnGATESAN, K., KUMAR, E., YUVARAJ, S., TANESH, P., \& KUMAR, A. (2020). An Approach for Remove Missing Values in Numerical and Categorical Values Using Two Way Table 
Marginal Joint ProbabilityInternational Journal of Advanced Science and Technology, 29(5), 27452756

15. Minje Jun, Deumji Woo and Eui-Young Chung (2012), “Partial Connection-Aware Topology Synthesis for On-Chip Cascaded Crossbar Network", IEEE Transactions On Computers, Vol. 61(1).

16. En-Jui Chang, Hsien-Kai Hsin, Shu-Yen Lin and An-Yeu (Andy) Wu (2014), "Path-CongestionAware Adaptive Routing with a Contention Prediction Scheme for Network-on-Chip Systems", IEEE Transactions On Computer-Aided Design Of Integrated Circuits And Systems, Vol. 33 (1), pp 231-245. 